\title{
WAVELET SHRINKAGE ADAPTIVE HISTOGRAM EQUALIZATION FOR MEDICAL IMAGES
}

\author{
Anbu Megelin star ${ }^{1)}$, Perumal Subburaj ${ }^{2)}$ \\ ${ }^{1)}$ Department of Electrical and Computer Engineering, Tamizhan College of Engineering and Technology, \\ Chenbagaramanputhoor, India, e-mail: anbumegelinstar@gmail.com \\ ${ }^{2)}$ Department of Electrical and Electronic Engineering, National Engineering College, Kovilpatti, TamilNadu, India \\ e-mail: subbu_neceee@yahoo.com
}

\begin{abstract}
Enhancement techniques play a major role in medical image processing, to improve the quality of raw images. This paper proposes a novel algorithm namely wavelet shrinkage adaptive histogram equalization (WSAHE) for medical image enhancement. This algorithm consists of four stages namely, decomposition of images using wavelet transform, application of adaptive histogram equalization on the approximation coefficients, application of shrinkage on the detailed coefficients and the reconstruction of image. Experiments show that the proposed method enhances the image brightness while preserving edges. Copyright (C) Research Institute for Intelligent Computer Systems, 2014. All rights reserved.
\end{abstract}

Keywords: adaptive histogram equalization, image enhancement, medical images, wavelet shrinkage.

\section{INTRODUCTION}

Image enhancement is a major part of image processing. Its objective is to process an image so that the result is more suitable than the original image for a specific application. In medical images, enhancement supports the surgeons for proper diagnosis, when the image is deteriorated by noise. Many medical image enhancement techniques such as histogram equalization (HE), filtering and frequency domain techniques have been studied. Histogram equalization and specification have been widely used in a gray scale image. Histogram equalization is a process which consists of generating an output image with a uniform histogram. In this method, the level of contrast enhancement cannot be controlled since the HE is a fully automatic algorithm without any parameter. They may suffer from the high complexity and blocking effect. Even though HE has advantages, it suffers from global brightness variation [1, 2]. In [9] Log based histogram modification method is described. It expands the values of pixel in an image while compressing higher level values. LHM provides better output than HE. It applies equalization on selective area pixel or pixels requiring equalization. The methods explained in $[10,11]$ derives local information from the neighboring windows with different sizes. The computational costs of this approach are very high because of the LSD and division operation in the sliding window with different sizes. This approach can over-enhance noise, resulting in an unnatural image. Filtering techniques [3, 4] were also used for image enhancement, but it has the drawback of noise amplification. In frequency domain techniques, 'Wavelet based technique $[5,6]$ is the dominant one, even though it has the advantage of noise elimination, it does not efficiently show the contours. To overcome all these problems, a new approach is introduced, which combines a wavelet shrinkage and adaptive equalization. The wavelet shrinkage is applied on the detailed coefficients of the decomposed image, in order to remove the noise present in the high frequency components and adaptive histogram equalization is applied on the approximation coefficients, to improve the contrast.

\section{METHOD}

In the discrete wavelet transform, an image signal can be analyzed by passing it through an analysis filter bank followed by a decimation operation as shown in Fig. 1. This analysis filter bank, which consists of a low pass and a high pass filter at each decomposition stage, is commonly used in image compression. When a signal passes through these filters, it is split into two bands. The low pass filter, which corresponds to an averaging operation, extracts the coarse information of the signal. The 
high pass filter, which corresponds to a differencing operation, extracts the detail information of the signal. The output of the filtering operations is then decimated by two.

A two-dimensional transform can be accomplished by performing two separate onedimensional transforms. First, the image is filtered along the $\mathrm{x}$-dimension and decimated by two. Then, it is followed by filtering the sub-image along the ydimension and decimated by two. Finally, we have split the image into four bands denoted by LL, HL, $\mathrm{LH}$ and $\mathrm{HH}$ after one-level decomposition.

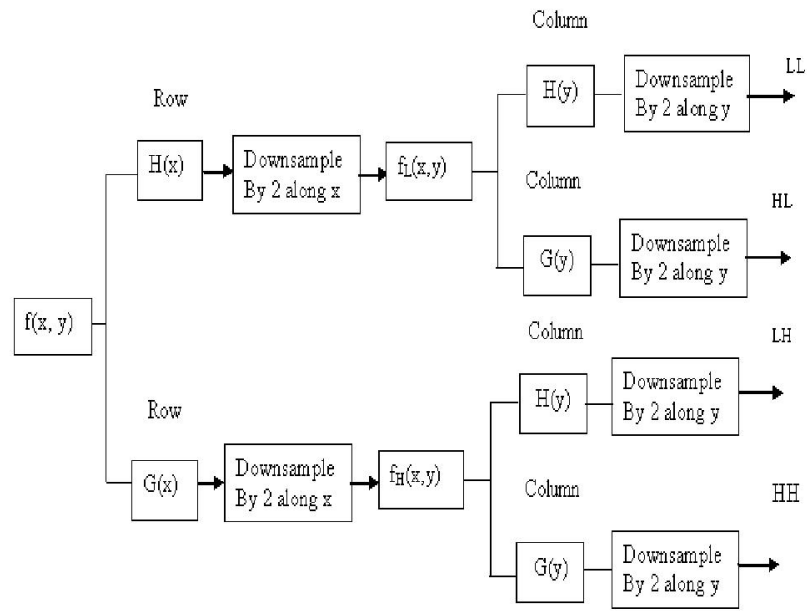

Fig. 1 - Wavelet transform.

The image $I(x, y)$ is decomposed using $\mathrm{db} 4$ wavelet transform for one level. It produces four sub images say LL, HL, LH and HH. Here LL is the approximation coefficient and it consists of low frequency components, $\mathrm{HL}, \mathrm{LH}$ and $\mathrm{HH}$ are detailed coefficients and it consists of high frequency components. The transformed coefficients are given by

$$
D_{[L L, H L, L H, H H]}=T(I(x, y)),
$$

To increase the contrast, the approximation coefficients are separated and perform the adaptive histogram equalization operation as given below.

$$
G_{L L}(p, q)=L\left(D_{L L}(p, q)\right),
$$

where L is the intensity transformation function, which transforms the input image into another image, that has a uniform histogram by using cumulative density function (CDF).

The detailed coefficients are separated and perform the shrinkage by soft thresholding. The soft threshold is obtained by

$$
T h_{[H L, L H, H H]}=\sigma_{[H L, L H, H H]} \sqrt{2 \log N_{[H L, L H, H H]}},
$$

Where

$$
\sigma=\sqrt{\frac{1}{N} \sum_{k=1}^{N}\left(x^{k}-\bar{x}\right)^{2}},
$$

where $\mathrm{N}$ represents the number of coefficients, $x^{k}$ is the coefficients and $\bar{x}$ is the mean value of the coefficients. Using the threshold, shrinkage is done on all detailed coefficients by

$$
O(p, q)=\left\{\begin{array}{c}
D-T h, D>T h \\
0,-T h \leq D \leq T h \\
D+T h, D \leq-T h
\end{array}\right.
$$

Finally the processed coefficients are reconstructed by taking inverse wavelet transform.

The wavelet inverse process is done based on the Fig. 2. First, all the four subbands at the coarsest scale are upsampled by a factor of two, and filter the subbands in each dimension. Then sum the four filtered subbands to reach the low-low subband at the next finer scale. This process is repeated until the image is fully reconstructed. In this paper the daubechis inverse wavelet process produces the enhance image.

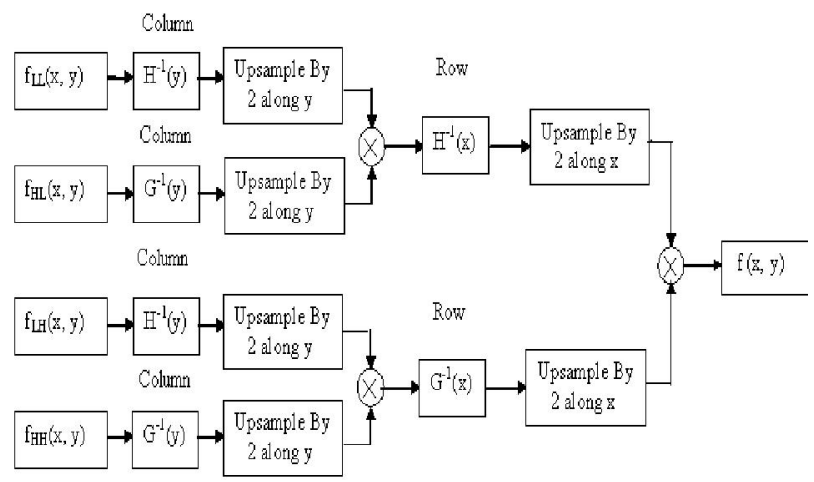

Fig. 2 - Inverse wavelet transform.

\subsection{MEDICAL IMAGE ENHANCEMENT ALGORITHM}

In this section, a histogram method is called Wavelet Shrinkage Adaptive histogram Equalization (WSAHE) is utilized to remove the unwanted noise from the input CT lung images. The overall block diagram of the noise removal system is presented in Fig. 3. In this noise removal algorithm, the wavelet transform is done one of significant performance to do the enhancement process. 


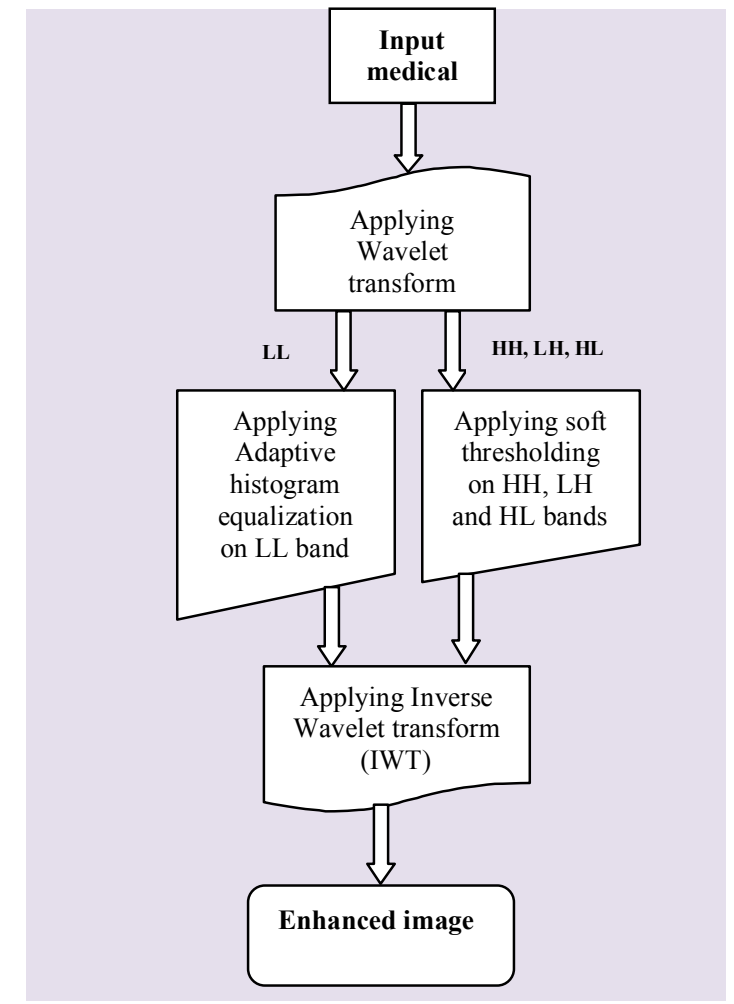

Fig. 3 - Block diagram of the noise reduction cum Enhanced algorithm.

\section{RESULTS}

Lung CT images are used to demonstrate the performance of this method. Biomedical image processing remains an important issue in medical and computer field. Since the emergence of digital acquisition in medical imaging, the data production is continuously growing. So there is a need for image enhancement which can be used for surgical planning.

The experimental results of the proposed medical image enhancement approach using daubechis wavelet transform are presented in this section. The proposed enhancement approach is programmed in Matlab (Matlab Version 7.12). The experimental results are carried out with diverse medical images which are collected from hospital. The medical image enhancement algorithm is done on lung computed tomography (CT) images. The enhanced images possess superior Peak Signal to Noise Ratio (PSNR), computation time and entropy for different size medical images of $512 \times 512$ and $128 \times 128$. The attained results illustrated the efficiency of proposed approach. As well, the comparative analysis and the robustness analysis are performed with the existing wavelet based enhancement algorithm.

\subsection{EVALUATION MATRICES}

The following formulas used to compute the evaluation metrics PSNR and entropy values are given as follows:

\section{Peak Signal to Noise Ratio (PSNR):}

The PSNR can measure the intensity difference between two images, it is well-known that it may fail to describe the visual perception quality of the image. The formula for PSNR value computation is,

$$
P S N R=10 \log _{10} \frac{E_{\max }^{2} \times I_{w} \times I_{h}}{\sum\left(I_{x y}-I_{x y}^{*}\right)},
$$

where $I_{w} \& I_{h} \rightarrow$ width and height of the denoised image; $I_{x y} \rightarrow$ original image pixel value at coordinate $(x, y) ; I_{x y}^{*} \rightarrow$ denoised image pixel value at coordinate $(x, y) ; E_{\max }^{2} \rightarrow$ largest energy of the image pixels.

\section{Entropy:}

Image Entropy is a quantity which is used to describe the 'business' of an image, i,e. the amount of information which must be coded for a enhancement algorithm. Also, it is the average amount of information contained in the enhanced image. The formula of the entropy is followed as

$$
\text { Entropy }=-\sum_{i} p_{i} \log _{2} p_{i},
$$

where $p_{i} \rightarrow$ probability that the differnce 2 adjacent pixels is equal to $i ; \log _{2} \rightarrow$ base 2 logarithm.

\subsection{EXPERIMENTAL RESULTS}

In order to estimate the effectiveness of the proposed method of wavelet shrinkage adaptive histogram equalization (WSAHE), it was compared against five existing methods: (i) Gabor filter, (ii) histogram equalization (HE), (iii) adaptive histogram equalization (AHE) [14], (iv) Contrast limiting adaptive histogram equalization (CLAHE) [13] and (v) Modified adaptive histogram equalization (MAHE). As mentioned above, Fig. 4 shows the image enhancement results for the proposed and existing methods.

\subsection{PERFORMANCE EVALUATION}

In this section, the performance of the proposed method is analyzed for different threshold levels. Here, the threshold level is varied for proposed approach in seven levels for sample 5 medical images. The effect of threshold $(T h)$ parameter on the enhancement performance in terms of PSNR is demonstrated in Fig. 5. Analyzing the figure, WSAHE method achieved the maximum PSNR value in threshold level 68 . 


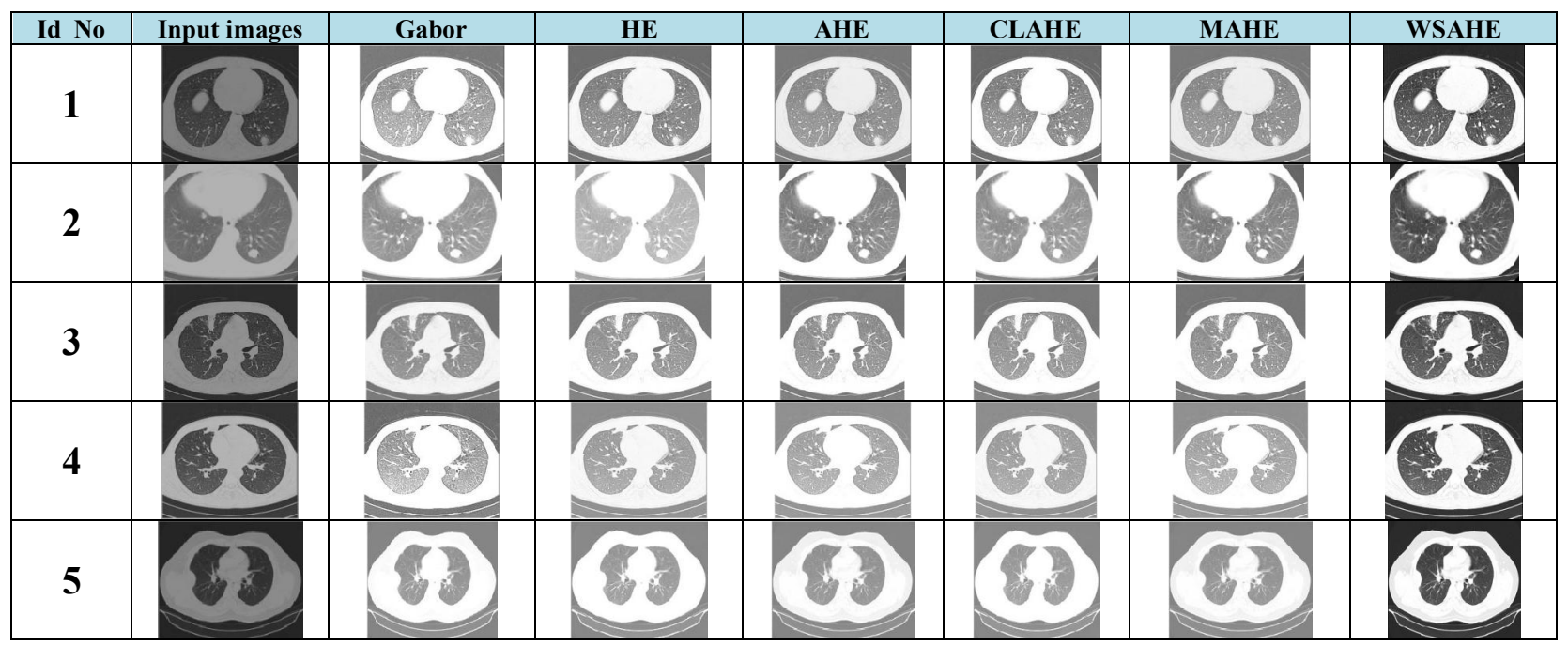

Fig. 4 - Image enhancement methods for proposed against existing methods.

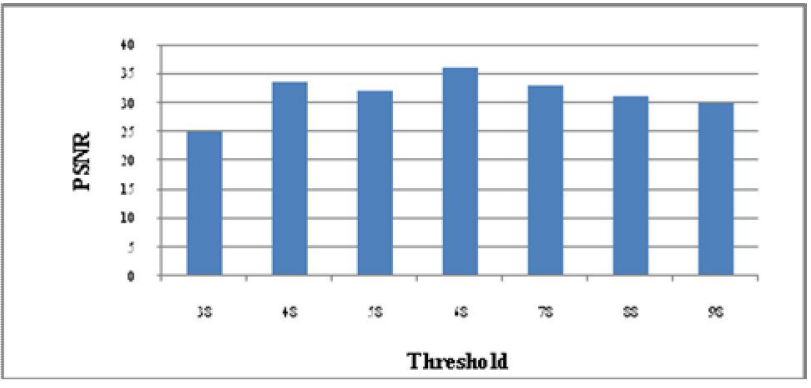

Fig. 5 - Effect of Threshold $(T h)$ on the enhancement performance.

\subsection{COMPARATIVE ANALYSIS}

In this section, the comparative analysis of the different enhancement systems are presented in terms of peak signal-to-noise ratio (PSNR), entropy and computation time. Table 1 illustrates the PSNR comparison for proposed against existing systems. Table 2 illustrates the entropy comparison for proposed against existing systems. In addition, different wavelets are applied with different thresholds level to find the effectiveness of proposed approach. Figures 6.1 to 6.5 show PSNR performance on different wavelets with proposed method (like daubechis, haar, biorthogonal, coifelts and symlets) for five CT images. From the results, it demonstrates that the daubechis based method acheived better perfromance than other wavelets. Table 3 lists the computation time performance of proposed against some existing systems (like Gabor, HE, AHE, CLAHE and MAHE) for five input images. For each system, the execution time is measured (in seconds) to prove the efficiency of proposed WSAHE system. From the table 3, it proves that the WSAHE based proposed system has acheieved better perfromance than existing systems.
Table 1. PSNR comparison for proposed against existing systems.

\begin{tabular}{|c|c|c|c|c|c|c|}
\hline \multicolumn{7}{|c|}{ PSNR (dB) } \\
\hline Input images & Gabor & HE & AHE & CLAHE & MAHE & WSAHE \\
\hline & 24.11 & 26.47 & 28.45 & 28.14 & 30.47 & 38.42 \\
\hline & 25.2 & 24.68 & 24.65 & 29.64 & 31.54 & 39.56 \\
\hline & 21.24 & 28.35 & 21.32 & 29.68 & 32.14 & 39.47 \\
\hline & 25.41 & 24.21 & 25.31 & 27.68 & 32.12 & 39.12 \\
\hline & & & & & & \\
\hline
\end{tabular}

Table 2. Entropy comparison for proposed against existing systems.

\begin{tabular}{|c|c|c|c|c|c|c|}
\hline \multicolumn{7}{|c|}{ Entropy } \\
\hline Input images & Gabor & HE & AHE & CLAHE & MAHE & WSAHE \\
\hline & 9.20 & 8.24 & 6.701 & 7.14 & 10.32 & 11.07 \\
\hline & 9.21 & 8.36 & 6.8 & 7.56 & 10.54 & 11.98 \\
\hline & 9.20 & 8.45 & 6.72 & 7.48 & 10.65 & 11.24 \\
\hline & 9.22 & 8.65 & 6.93 & 7.68 & 10.47 & 11.36 \\
\hline & 9.23 & 8.45 & 6.47 & 7.48 & 9.78 & 10.98 \\
\hline
\end{tabular}




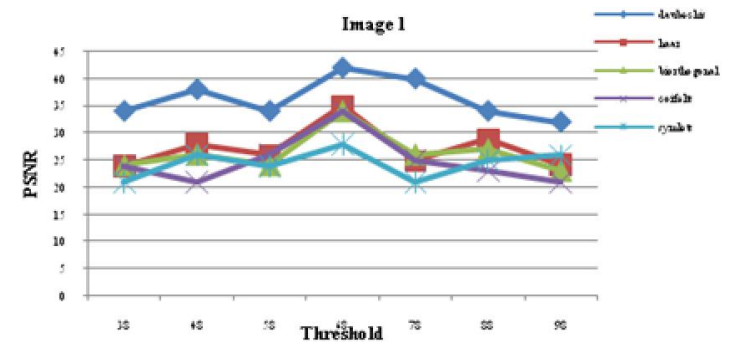

Fig. 6.1 - Wavelet based PSNR comparison in different thresholds for image 1.

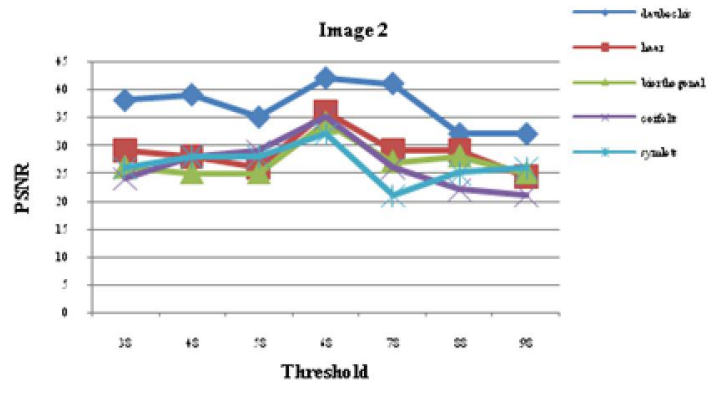

Fig 6.2 - Wavelet based PSNR comparison in different thresholds for image 2 .

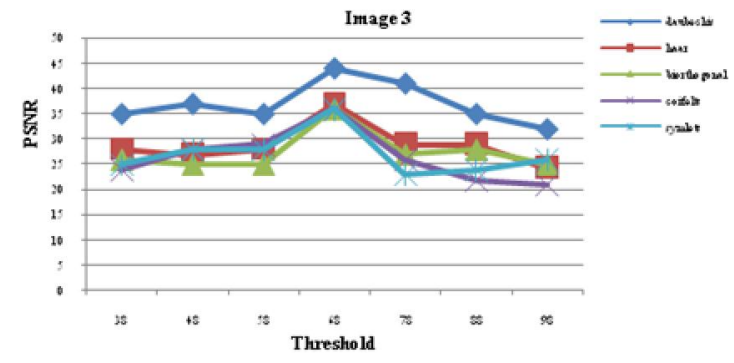

Fig 6.3 - Wavelet based PSNR comparison in different thresholds for image 3 .

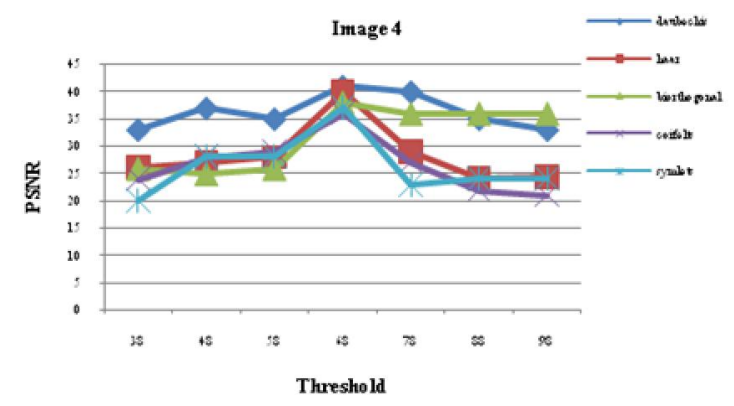

Fig 6.4 - Wavelet based PSNR comparison in different thresholds for image 4 .

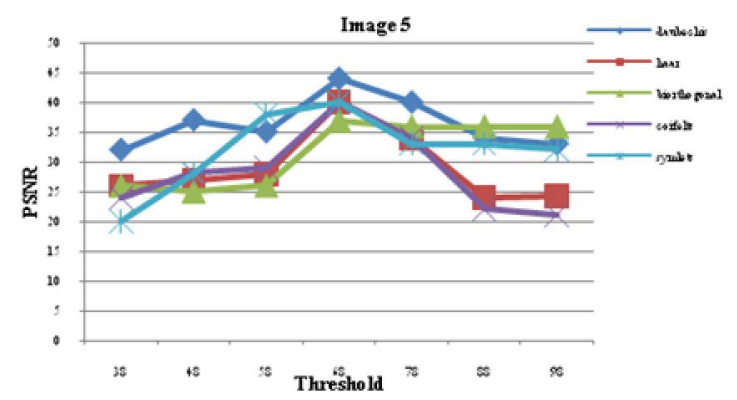

Fig 6.5 - Wavelet based PSNR comparison in different thresholds for image 5 .
Table 3.Time taken performance for various methods.

\begin{tabular}{|l|c|}
\hline \multicolumn{1}{|c|}{ Method Name } & Time taken (sec) \\
\hline Gabor Filter & 0.940 \\
\hline Histogram Equalization & 0.655 \\
\hline Adaptive Histogram Equalization & 0.815 \\
\hline $\begin{array}{l}\text { Contrast Limiting Adaptive } \\
\text { Histogram Equalization }\end{array}$ & 0.937 \\
\hline $\begin{array}{l}\text { Modified Adaptive Histogram } \\
\text { Equalization }\end{array}$ & 0.981 \\
\hline WSAHE(proposed) & 1.138 \\
\hline
\end{tabular}

\section{CONCLUSION}

A novel method for medical image enhancement based on the wavelet transform, shrinkage and histogram equalization was presented. The experimental results showed that the method has produced good quantitative and qualitative results in comparison to other technique such as adaptive histogram equalization and multiscale adaptive histogram equalization.

\section{REFERENCES}

[1] T. Kim, J. Paik, Adaptive contrast enhancement using gain-controllable clipped histogram equalization, IEEE Transactions on Consumer Electronics, (54) 4 (2008), pp. 1803-1810.

[2] M. Sundaram, A. Ramar, N. Arumugam, G. Prabin, Histogram modified local contrast enhancement for mammogram images, Applied Soft Computing, (11) 8 (2007), pp. 5809-5816.

[3] A. Polesel, G. Ramponi, V. J. Mathews, Image enhancement via adaptive unsharp masking, IEEE Transactions on Image Processing, (9) 3 (2000), pp. 505-510.

[4] C. C. Yang, A modification for the maskfiltering approach by superposing anisotropic derivatives in an image, International journal for Light and Electron Optics, (122) 18 (2011), pp. 1684-1687.

[5] P. Heinlein, P. Drexl, W. Schneider, Integrated wavelets for enhancement of microcalcifications in digital mammography, IEEE Transactions on Medical Imaging, (22) 3 (2003), pp. 402-413.

[6] H. D. Cheng, R. Min, M. Zhang, Automatic wavelet base selection and its application to contrast enhancement, Signal Processing, (90) 4 (2010), pp. 1279-1289.

[7] The Daubechies D4 Wavelet Transform, http://www.bearcave.com/misl/misl_tech/wavel ets/daubechies/.

[8] J. Stark, Adaptive image contrast enhancement using generalizations of histogram equalization, IEEE Transaction on Image Processing, (9) 5 (2000), pp. 889-896. 
[9] Z. Rahman, Retinex processing for automatic image enhancement, Journal of Electronic Imaging, (13) 1 (2004), pp. 100-110.

[10] K. Schutte, Multi-scale adaptive gain control of IR images, in Proceedings of SPIE, (3061) (1997), pp. 906-914.

[11] S. Cvetkovic, J. Schirris, P. de With, Locallyadaptive image contrast enhancement without noise and ringing artifacts, in Proceedings of ICIP, (3) (2007), pp. 551-560.

[12] V. Gopalakrishnan, Y. Hu, D. Rajan, Salient region detection by modeling distributions of color and orientation, IEEE Transactions on Multimedia, (11) 5 (2009), pp. 892-905.

[13] K. Zuiderveld, Contrast Limited Adaptive Histograh Equalization, in Book Graphic gems IV, San Diego: Academic Press Professional, 1994, pp. 474-485.

[14] S. M. Pizer, E. P. Amburn, J. D. Austin, et al, Adaptive histogram equalization and its variations, Computer Vision, Graphics, and Image Processing, (39) 3 (1987), pp. 355-368.

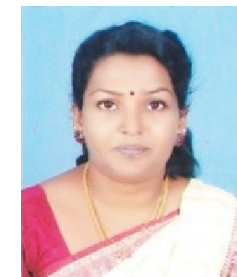

Anbu Megelin star received her B.E. in 1998 at the Indian Engineering College, Vadakangulam, M.E. (Pr Control \& Instrumentation) at Annamalai University, and Ph.D proceeding (Anna University Chennai). Her present position is Assistant Professor, ECE Dept, Tamizhan College of Engineering \& Technology. Her research area: Digital Image Processing, soft computing.

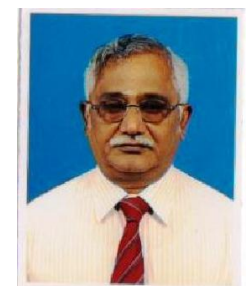

Dr P. Subburaj received his B.E. in 1977 at Thiagarajar College of Engineering, Madurai, M.E. (Power System) at Thiagarajar College of Engineering, Madurai, and Ph.D. at Anna University Chennai. His present position is Professor at EEE Dept, National Engineering College. His research area: Power System Economics, soft computing, High Voltage Engineering. 